\title{
QUISTE DE INCLUSIÓN EPIDÉRMICO VULVAR COMO COMPLICACIÓN TARDÍA DE CIRCUNCISIÓN RITUAL
}

\author{
Jorge Andrés Robert S. ${ }^{1}$, Roberto Yazigi I. ${ }^{1}$, Paolo Ricci A. ${ }^{1}$, Antonio Piottante B. ${ }^{2}$ \\ ${ }^{1}$ Departamento de Obstetricia y Ginecología, ${ }^{2}$ Departamento de Anatomía Patológica, Clínica Las Condes.
}

\section{RESUMEN}

Presentamos un caso de quiste de inclusión epidérmico, como complicación tardía, en una mujer africana con antecedente de mutilación genital tipo II o clitoridectomía total, durante su infancia.

\section{PALABRAS CLAVE: Quiste de inclusión epidérmico vulvar, quiste para-clitorideano, quiste vulvar, pseudohipertrofia de clítoris}

\section{SUMMARY}

We report a case of epidermal inclusion cyst as a late complication in an African woman with history of ritual genital mutilation type II or total excision during childhood.

\section{KEY WORDS: Vulvar epidermoid inclusion cyst, para-clitoris cyst, vulvar cyst, pseudo-hypertrophy of the clitoris}

\section{INTRODUCCIÓN}

Los tumores benignos y malignos constituyen el $22 \%$ de la patología vulvar. Entre los más frecuentes se encuentran: quiste de inclusión epidérmico, léntigo, quiste por obstrucción del conducto excretor de la glándula de Bartholino, carcinoma in situ, nevus melanocítico, acrocordón, quiste mucoso, hemangioma, hiperpigmentación post-inflamatoria, queratosis seborreica, várices, hidroadenomas, verrugas y carcinoma de células basales. Entre los menos frecuentes destacan los neurofibromas, implantación de tejidos ectópicos, siringomas y abscesos (1). La gran variedad de tumores que pueden afectar la vulva, en la mayoría de los casos hace indispensable la biopsia para confirmar un diagnóstico.

Aunque infrecuentes, entre los diagnósticos diferenciales se encuentran los quistes como complicación de una circuncisión del clítoris. El origen de la paciente, y el antecedente de la exposición a esta práctica ritual durante la etapa infantil, lo que aún ocurre en algunos países del continente africano, debe hacer sospechar esta posible etiología.

Se presenta un caso de quiste de inclusión epidérmico vulvar como complicación tardía de una circuncisión genital femenina.

\section{Caso clínico}

Mujer de 38 años, originaria de Eritrea, multípara de 2 cesáreas, que consulta en octubre del 2009 por aumento de volumen en la región vulvar de varios años de evolución. Relata que no le produce interferencia con la micción ni con la actividad sexual, sin embargo tiene molestias ocasionadas por el volumen del tumor. No se registran otros antecedentes médicos de importancia. El examen físico general es normal. Al examen ginecológico 
destaca en la región supra-clitorídea, un aumento de volumen ovalado, de aproximadamente $3 \times 2 \times$ $2 \mathrm{~cm}$, quístico e indoloro a la palpación (Figura 1). No se visualizó el clítoris, en cuyo lugar estaba el tumor descrito. Los labios menores y mayores eran de aspecto normal. La observación de la vagina y del cuello uterino no reveló alteraciones.

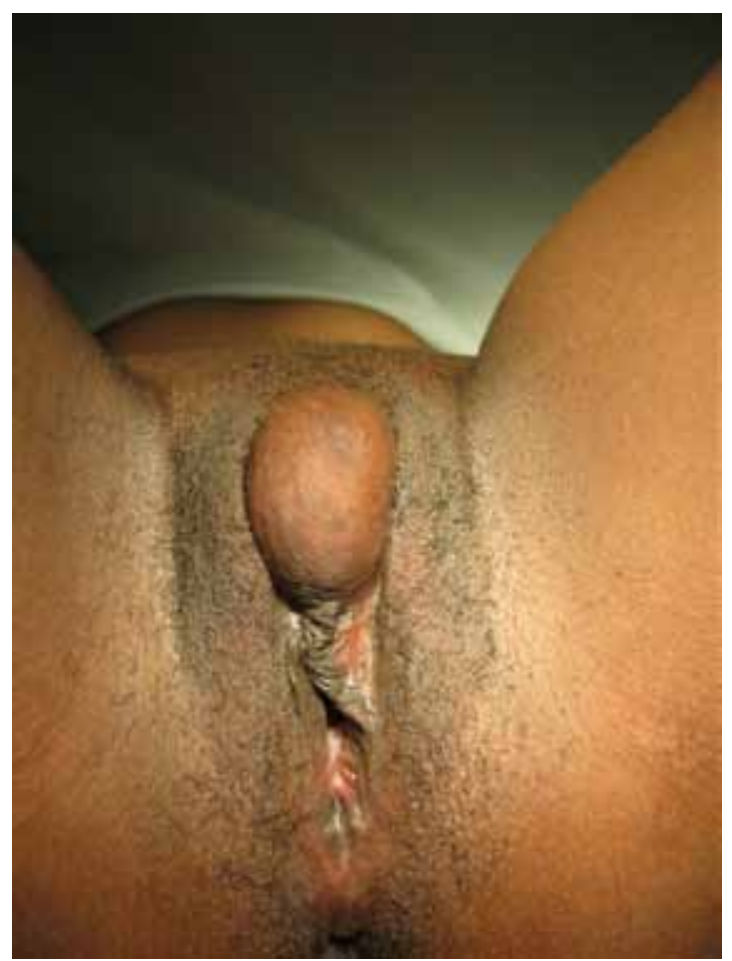

Figura 1. Tumor observado en la posición normal del clítoris.

La ecografía ginecológica confirmó la presencia de útero y ovarios de aspecto normal. El tumor demostró un contenido homogéneo denso semejante al de los quistes endometriósicos y sin evidencia de flujo sanguíneo en su interior (Figura 2).

Con anestesia regional y en posición ginecológica, se realizó la extirpación completa del tumor, disecando con tijeras el plano entre la superficie del tumor y el celular subcutáneo/piel (Figuras 3 y 4). La biopsia intra-operatoria demostró que se trataba de un quiste de inclusión epidérmico, sin atipías, lo que fue confirmado en la biopsia diferida (Figura 5). Se resecó la piel redundante y se suturó con monocril 5/0 subcuticular (Figura 6).

Concluida la cirugía y con el diagnóstico anátomo-patológico de quiste de inclusión epidérmica y la confirmación de ausencia de clítoris sumado al

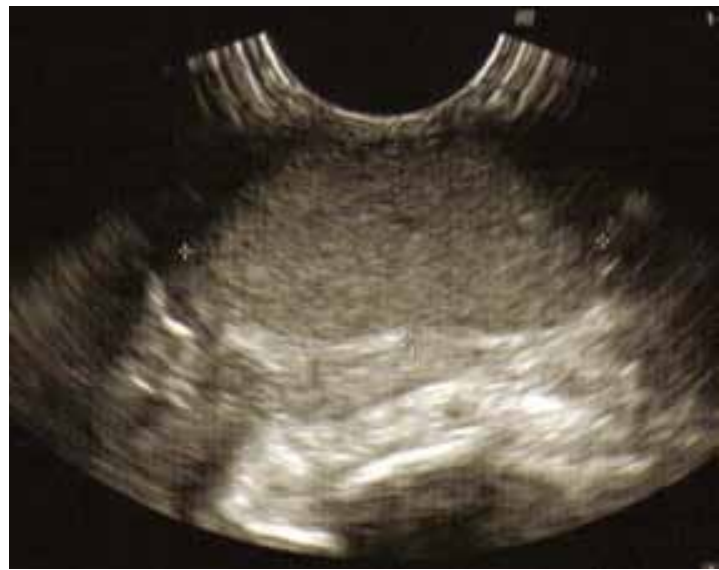

Figura 2. Visión ecográfica del tumor.

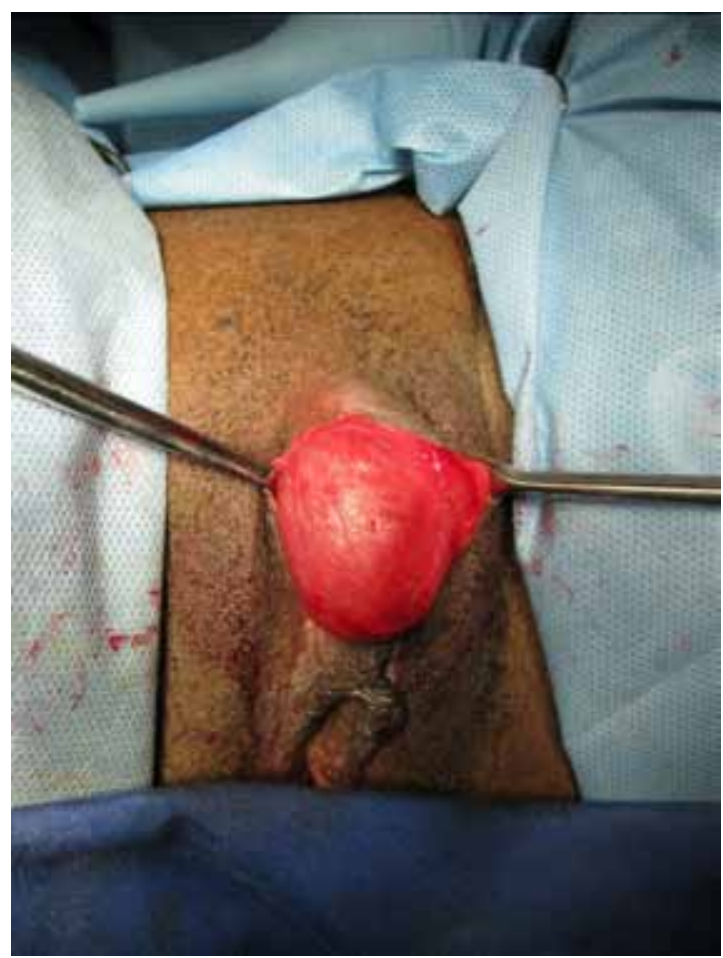

Figura 3. Se observa el quiste durante su extirpación.

origen étnico de la paciente, se le preguntó directamente por el antecedente de clitoridectomía ritual durante la infancia, lo cual fue confirmado. Fue dada de alta al día siguiente y controlada a los catorce días, con un examen de la zona operatoria normal. 


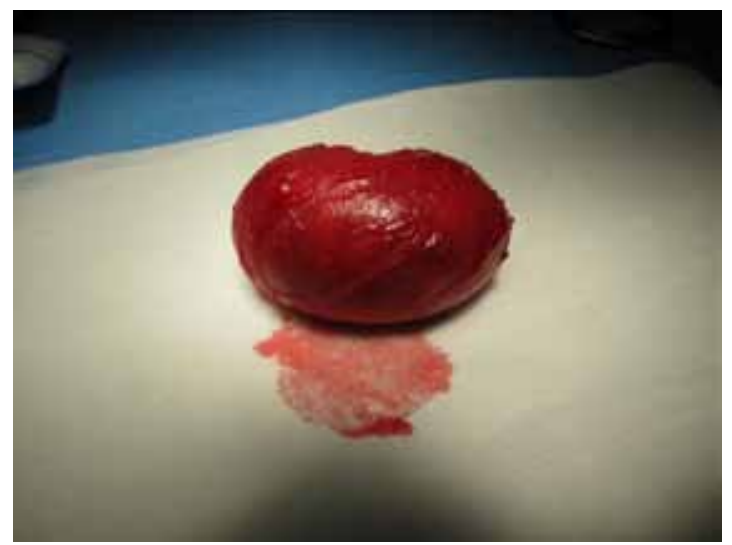

Figura 4. Detalle del quiste una vez extirpado.

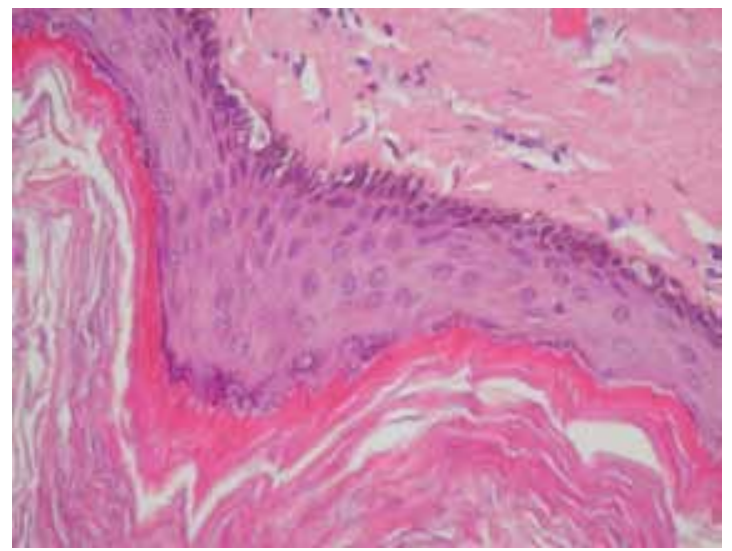

Figura 5. Quiste revestido por epitelio escamoso maduro, con áreas de acantosis (engrosamiento) y queratinización acentuada (en la cavidad se ven numerosas escamas córneas desprendidas).

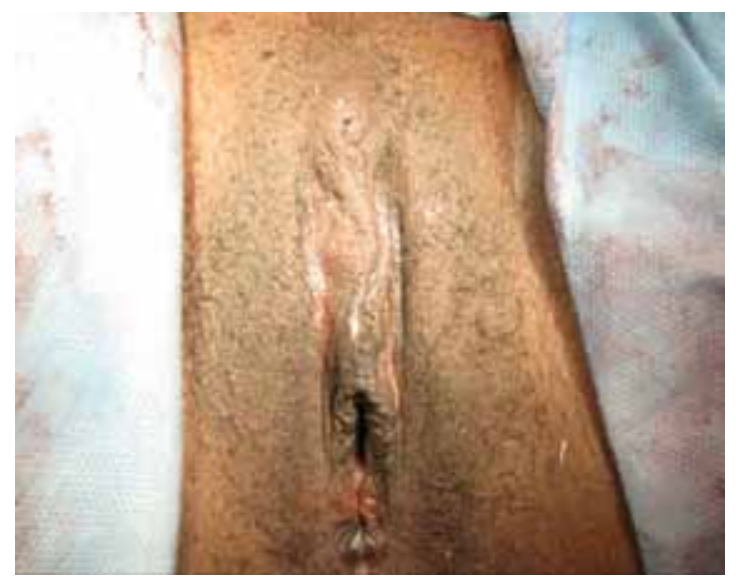

Figura 6. Resultado final.

\section{DISCUSIÓN}

Aunque inhabituales, se han reportado algunos casos de quistes de inclusión epidérmicos como complicación tardía después de una mutilación genital femenina (2-4). La mayoría de estos informan casos que corresponden a mujeres con antecedente de circuncisión del clítoris, efectuada como costumbre ritual lo que se conoce como "sunna" (del musulmán, tradición o deber), en que se reseca parte o todo el clítoris. Menos frecuente, algunos casos corresponden a la llamada "circuncisión simbólica", en la que no se remueve el clítoris y sólo se hace sangrar "simbólicamente". En estas últimas mujeres no se genera un daño a largo plazo y el clítoris queda intacto. La gran mayoría de los reportes de la literatura corresponden a mujeres con antecedente de este tipo de mutilación ritual, por lo que se describe la extracción del quiste y la preservación del clítoris. En el caso que reportamos, no se encontró el clítoris, por lo que se deduce que fue mutilado en conjunto con el "capuchón" o prepucio protector, tratándose de una mutilación completa o clitoridectomía.

La clitoridectomía constituye tal vez la práctica menor de este ritual al que se ven forzadas unas 6000 mujeres cada día en el mundo $(5,6)$. Los casos más radicales constituyen la llamada infibulación o circuncisión faraónica (7). En estas mujeres se extirpa el clítoris, labios menores y parte de los mayores. Luego se suturan ambos lados de la vulva y se dejan unidos, permaneciendo un pequeño orificio para la salida de la orina y la menstruación. Las piernas, permanecen vendadas y unidas entre sí por un período de tiempo prolongado para facilitar la cicatrización.

La mutilación genital femenina constituye una práctica ritual, forzada, que es realizada aún en por lo menos 28 países, mayoritariamente africanos, en niñas entre los 2 y 7 años de edad. Es una práctica social ritual y no religiosa, cuya persistencia en muchos lugares pretende ser explicada dando una fundamentación religiosa. El objetivo es evitar que las mujeres tengan placer en la relación sexual, para asegurar que no "serán infieles" (8). Hoy la práctica de esta mutilación es ilegal en muchos países de África, donde a pesar de esto, continúa como una costumbre frecuente. Sin embargo, algunos reportes indican que a partir de la década del 40 , con la creación de leyes en su contra, se comenzaron a realizar las denominadas mutilaciones "intermedias", que son extirpaciones de diferente cuantía entre la sunna y la infibulación faraónica.

La mutilación genital femenina es más frecuente en África, aunque se ha extendido a países vecinos 
y musulmanes de Asia. Los países en que se observa esta práctica son Egipto, Etiopía Eritrea, Sierra Leona, Somalia, Sudán, Benin, Djibouti, Chad, Gambia, Guinea, Kenia, Liberia, Mali, Nigeria, Burkina Faso y Togo, entre otros, y con una menor frecuencia en Malasia e Indonesia. Se cree que por lo menos 130 millones de mujeres han sido sometidas a esta práctica en el mundo (ONU-UNICEF) (8).

Otra forma de clasificación de la mutilación genital femenina ritual, ampliamente utilizada en las publicaciones científicas, es la numérica: la tipo I o remoción sólo del prepucio y/o una pequeña parte del clítoris; la tipo II o remoción completa del clítoris; la tipo III o extirpación del clítoris y labios menores y la tipo IV que corresponde a la infibulación faraónica. Los escasos reportes en la literatura corresponden a quistes de inclusión epidérmica en mujeres con mutilación tipo I. El caso que se presenta, corresponde a un quiste de inclusión en una mujer con mutilación genital de tipo II.

Los síntomas y signos que se describen como causa de la consulta médica en las mujeres que tienen estos quistes de inclusión vulvar son dolor, prurito y aumento de volumen localizado, que según el tamaño, puede interferir en la deambulación o en la actividad física (9). Con menos frecuencia se han descrito mujeres con descarga intermitente o esporádica de flujo desde la zona de cicatriz. También se han descrito casos de ruptura espontánea (7). En la década del setenta se informó un caso infectado con actinomyces israelii (10).

El signo que identifica a estos quistes es la presencia de implantación dermoide en el estudio de anatomía patológica en el quiste (7). En su etiopatogenia, estos quistes se producen como complicación tardía a una mutilación efectuada cruentamente, en la clandestinidad, con pocas medidas higiénicas y con instrumental inapropiado para procedimientos quirúrgicos. Muchas de las mujeres expuestas a estas extirpaciones mueren por complicaciones infecciosas inmediatas como el tétano.

Los quistes de inclusión epidérmica pueden ser adquiridos o congénitos. Los adquiridos se pueden observar en la vulva, incluyendo el clítoris, como resultado de la implantación profunda de un trozo de epidermis, debido a una herida penetrante o una incisión quirúrgica. Los congénitos corresponden a los pilonidales, que son producto del cierre incompleto de los pliegues genitales donde habitualmente se desarrollan (11).

Debemos mencionar que también se han descrito estos quistes de inclusión epidérmica vulvar en mujeres sin antecedente de circuncisión ritual, probablemente asociados a traumas (12). En una revisión de 40 casos de quistes benignos de vulva, el $25 \%$ correspondió a quistes de inclusión epidérmica (13). Algunos casos de quistes contienen en su interior calcificaciones como parte de la respuesta inflamatoria (14).

Finalmente, debemos mencionar que a simple vista, los quistes de inclusión que afectan al clítoris pueden simular una hipertrofia de éste $(2,15)$. Otros diagnósticos diferenciales del quiste de inclusión clitorideano que debemos tener presente principalmente en mujeres jóvenes, son la hipertrofia verdadera de clítoris por hiperplasia adrenal y la neurofibromatosis (16).

\section{CONCLUSIONES}

Entre los diagnósticos diferenciales de tumor vulvar debemos considerar el quiste de inclusión epidérmico. Aunque es poco frecuente, debe sospecharse si existen antecedentes de circuncisión ritual, cirugías previas y/o traumas, que puedan haber facilitado la inclusión y secuestro en profundidad de epidermis. La circuncisión ritual, que puede comprender desde la clitoridectomía hasta la amputación completa de labios menores y parcial de los labios mayores, que en su grado mayor es conocida como infibulación faraónica, es una práctica de violencia contra mujeres jóvenes que, a pesar de ser ilegal, aun se realiza en algunos países de África.

\section{BIBLIOGRAFÍA}

1. Hood AF, Lumadrue J. Benign vulvar tumors. Dermatol Clin 1992;10(2):371-85.

2. Rizk DE, Mohammed $\mathrm{KH}$, Joshi SU, Al-Shabani AY, Bossmar TR. A large clitoral epidermoid inclusion cyst first presenting in adulthood following childhood circumcision. J Obstet Gynaecol 2007;27(4):445-8.

3. Yoong WC, Shakya R, Sanders BT, Lind J. Clitoral inclusion cyst: a complication of type I female genital mutilation. J Obstet Gynaecol 2004;24(1):98-9.

4. Rouzi AA, Sindi O, Radhan B, Baáqeel H. Epidermal clitoral inclusion cyst after type I female genital mutilation. Am J Obstet Gynecol 2001;185(3):569-71.

5. Kelly E, Hillard PJ. Female genital mutilation. Curr Opin Obstet Gynecol 2005;17(5):490-4.

6. Darkenoo E. 6000 girls at risk every day. Female genital mutilation, although illegal, is still widely practiced. Womens Health Newsl 1993;20:10-1.

7. Hanly MG, Ojeda VJ. Epidermal inclusion cysts of the clitoris as a complication of female circumcision and pharaonic infubulation. Cent Afr $\mathrm{J}$ Med 1995;41(1):22-4.

8. Utz-Billing I, Kentenich $\mathrm{H}$. Female genital mutilation: an injury, physical and mental harm. J Psychosom Obstet Gynaecol 2008;29(4):225-9.

9. Kroll GL, Miller L. Vulvar epitelial inclusion cyst as a late complication of chilhood female traditional genital 
surgery. Am J Obstet Gynecol 2000; 183(2):509-10.

10. McElroy JY, Gorens ME, Jackson LN, Stigger D, Becker T, Sheiner E. Actinomyces israelii may produce vulvar lesions suspicious for malignancy. Infect Dis Obstet Gynecol 2006;2006:48269.

11. Charte Alegre ME, Bono Ariño A, Berné Manero JM, Vera Álvarez J, Sanz Vélez JI. Intraescrotal epidermal inclusion cysts. Actas Urol Esp 2000;24(9):771-3.

12. Oonk MH, Pelinck MJ. Diagnostic image (389). A woman with a multicyst vulvar mass. Ned Tijdschr Geneeskd 2008;152(37):2015.

13. Kondi-Pafiti A, Grapsa D, Papakonstantinou K, KairiVassilatou E, Xasiakos D. Vaginal cysts: acommon pathologic entity revisited Clin Exp Obstet Gynecol 2008;35(1):41-4.

14. Al-Maghrabi J, Kanaan $\mathrm{H}$, Bondagji N. Postcircumcision epidermoid inclusion cyst of the vulva containing multiple stones. Int J Gynaecol Obstet 2005;90(2):155-6.

15. Egger $\mathrm{H}$. Epidermal inclusion cyst presenting as hypertrophy of the clitoris 14 years after vulvar trauma. Geburtshilfe Frauenheilkd 1973;33(8):672-3.

16. Cost NG, Sanchez FS, Weinberg AG, Koral K, Baker LA. Neurofibromatosis presenting as painless clitoromegaly. Urol J 2009;6(3):220-2. 